\title{
Correction to: Dehydroepiandrosterone sulphate (DHEAS) concentrations stringently regulate fertilization, embryo development and IVF outcomes: are we looking at a potentially compelling 'oocyte-related factor' in oocyte activation?
}

\author{
Bindu N. Chimote ${ }^{1} \cdot{\text { Natachandra M. } \text { Chimote }^{2}}^{2}$ \\ Published online: 4 May 2021 \\ (C) Springer Science+Business Media, LLC, part of Springer Nature 2021
}

\begin{abstract}
Purpose Erratic oocyte-activation affects fertilization and embryo development. Dehydro-epiandrosterone sulphate (DHEAS) is present in theca/cumulus-granulosa cells, regulates the same calcium-pumps that cause calcium-oscillations in mice and its levels are altered in women with no or low fertilization rates. Yet no study has explored correlation of DHEAS with oocyte-activation. We proposed to implicate DHEAS as an oocyte-related factor in oocyte-activation by demonstrating that rectification of deviated (both lower/and higher than normal) DHEAS concentrations to normal post-treatment improves fertilization, embryo development rates. Method Prospective Closed-Cohort. Recruited $n=750$ (150 women/subgroup) in previously classified Low(A), Average/ Control(B), High(C) D3-serum DHEAS groups. 50\% women in both A and C groups received 3-months exposure to oral DHEAS and Metformin respectively. Also measured Follicular-fluid DHEAS levels. Compared embryologic, clinical outcomes: DHEAS untreated (A1) vs. treated (A2); metformin untreated (C1) vs. treated (C2) and A1/A2/C1/C2 against normal-control-B group. Also compared serum vs. FF-group results.

Results Significantly improved embryologic, clinical parameters in treated $\mathrm{A} 2 / \mathrm{C} 2$ compared to untreated $\mathrm{A} 1 / \mathrm{C} 1$ subgroups respectively. Post-treated improved parameters in $\mathrm{A} 2 / \mathrm{C} 2$ were comparable with, whereas untreated $\mathrm{A} 1 / \mathrm{C} 1$ sub-groups had significantly lower values than, normal-control B-group. Parameters differed significantly between Low, Medium, High FFDHEAS groups. Results in serum vs. FF: A1 vs. LowFF, C1 vs. HighFF and A2/C2/B vs. MediumFF were comparable. Odds of fertilization, live-births increased in post-treatment A2/C2 subgroups. Fertilization rates strongly correlated with FF-DHEAS.

Conclusion Rectification of deviated DHEAS levels post-treatment significantly improves outcomes, comparable with those exhibited by normal-control DHEAS thresholds. DHEAS is the most promising endogenous oocyte-related factor influencing embryologic, clinical IVF outcomes possibly by affecting oocyte-activation.
\end{abstract}

A Correction to this paper has been published: https://doi.org/ $10.1007 / \mathrm{s} 10815-021-02215-\mathrm{z}$

Correction to: Journal of Assisted Reproduction and Genetics (2021) 38:193-202.

https://doi.org/10.1007/s10815-020-02001-3

The online version of the original article can be found at https://doi.org/10. 1007/s10815-020-02001-3

Bindu N. Chimote

bindunm10@yahoo.com; https://orcid.org/0000-0001-5387-9360

Natachandra M. Chimote

nmchimote@yahoo.co.in
The abstract in original article unfortunately was not included.

Publisher's note Springer Nature remains neutral with regard to jurisdictional claims in published maps and institutional affiliations.
1 IVF Embryology Laboratory, Vaunshdhara Fertility Centre, 9, Dr. Munje Marg, Congress Nagar, Nagpur, Maharashtra, India

2 Department of Reproductive Endocrinology, Vaunshdhara Fertility Centre, 9, Dr. Munje Marg, Congress Nagar, Nagpur, Maharashtra, India 